\title{
XAFS Study of Local Structure Change in Perovskite Titanates
}

\author{
K. Sato ${ }^{1}$, T. Miyanaga ${ }^{1}$, S. Ikeda $^{1}$ and D. Diop ${ }^{2}$ \\ ${ }^{1}$ Department of Materials Science and Technology, Faculty of Science and Technology, Hirosaki University, Aomori 036-8561, Japan \\ ${ }^{2}$ Department of Physics, Faculty of Science and Technique, University Anta Cheikh Diop of Dakar, Dakar, Senegal
}

Received June 26, 2003; accepted in revised form June 23, 2004

PACS numbers: 02.40.- $\mathrm{k}, 12.39 . \mathrm{Dc}, 14.80 . \mathrm{Hv}$

\begin{abstract}
The Ti K-edge EXAFS for $\mathrm{PbTiO}_{3}$ measured in the temperature range including the critical temperature $(763 \mathrm{~K})$ due to the phase transition from tetragonal to cubic phase is discussed from the local point of view. Three kinds of atomic pairs, shorter Ti-O(1), medium Ti-O(2) and longer Ti-O(3) were analyzed independently. The interatomic distance of $\mathrm{Ti}-\mathrm{O}(3)$ changes at $T_{c}$ discontinuously and shows a "displacive" type transition. On the other hand, EXAFS shows that the lattice distortion remains even in cubic phase, which indicates "order-disorder" type transition. The peak intensity at the pre-edge region in XANES, which indicates the distortion of Ti atoms along the $c$-axis of the oxygen octahedron, corresponds to the distortion obtained from EXAFS analysis.
\end{abstract}

\section{Introduction}

X-ray absorption fine structure (XAFS) has become a technique to study local structures as a complementary technique to diffraction methods which are based on long-range order [1]. $\mathrm{PbTiO}_{3}$ has long been considered as "displacive" type ferroelectrics: The perovskite $\mathrm{PbTiO}_{3}$ shows tetragonal structure at $T<T_{c}(=763 \mathrm{~K})$ (lattice parameters $a=b=3.89 \AA$ and $c=4.15 \AA$ at $300 \mathrm{~K}$ [2]) and cubic structure at $T>T_{c}(a=b=c=3.905 \AA$ at $773 \mathrm{~K})$. On the other hand, if the local distortion does not change with temperature across $T_{c}$ the transition is "order-disorder" type. In this case, only the orientations of the distortion within a small domain change with temperature; the orientation is arranged along the preferable direction below $T_{c}$, but it is disordered above $T_{c}$.

The local structural change of $\mathrm{PbTiO}_{3}$ has been extensively studied by Sicron et al. using XAFS of both $\mathrm{Pb} \mathrm{L}_{\mathrm{III}}$ and $\mathrm{Ti} \mathrm{K}$ edges [3, 4]. These results show that an essential element of "order-disorder" is present even in this normally pure ferroelectric crystal, which displays a soft mode and a dielectric constant of typical "displacive" type ferroelectrics. Recently, a more detailed study by $\mathrm{Pb} \mathrm{L}_{\mathrm{III}}$ edge EXAFS for $\mathrm{PbTiO}_{3}$ has been carried out [5] where three kinds of atomic pairs $\mathrm{Pb}-\mathrm{O}(1,2,3)$ are analyzed independently. Shorter atomic pairs of $\mathrm{Pb}-\mathrm{O}(1,2)$ show "orderdisorder" type behavior, on the other hand, the longest $\mathrm{Pb}-\mathrm{O}(3)$ shows "soft mode" behavior [5].

In this paper, we study the local structural change around $\mathrm{Ti}$ atoms in $\mathrm{PbTiO}_{3}$ crystal through $T_{c}$ in more detail. Actually, there exist three types of Ti-O pairs by tetragonal distortion; longer and shorter interatomic distances along the $c$-axis and a medium Ti-O pairs perpendicular to the $c$-axis. Our results show that the local distortion remains above $T_{c}$ in which $\mathrm{PbTiO}_{3}$ shows cubic phase.

\section{Experimental and Data Analyses}

The powder sample of $\mathrm{PbTiO}_{3}(99.99 \%)$ was purchased from Soeakwa Co. Ltd. and was checked by X-ray powder diffraction to assure its crystal structure. The powder was mixed with $\mathrm{BN}$ and was dry pressed for producing a pellet that could be easily handled and placed within the furnace. X-ray absorption spectra of Ti Kedge (4.95 keV) were measured at BL9A of Photon Factory (KEK) in transmission mode. A $\mathrm{Si}(111)$ double crystal monochrometer was used. The temperature range for measurement was from $300 \mathrm{~K}$ to $923 \mathrm{~K}$.

The EXAFS analysis was performed according to XANADU code [6] and it is given only briefly here. The normalized EXAFS interference function, $\chi(k)$, was multiplied by a Hamming window function to reduce the ripples in the Fourier-transformed spectra in $r$-space. The $k$-range for the Fourier transform was from 2.95 to $8.8 \AA^{-1}$.

\section{Results and Discussion}

We discuss here the result for Ti K-edge XAFS. Figure 1 shows the EXAFS oscillation functions, $k^{2} \chi(k)$, of Ti K EXAFS for $\mathrm{PbTiO}_{3}$ crystal at various temperatures obtained by transmission measurement. Although the signal in the transmitted ionization chamber is not so large due to the absorption by $\mathrm{Pb}$ atoms, satisfactory data quality has been obtained up to $8.5 \AA^{-1}$ for EXAFS analyses even at high temperatures. As the temperature increases the amplitude of the EXAFS function decreases gradually. Figure 2 shows the Fourier transforms of Ti K EXAFS for $\mathrm{PbTiO}_{3}$ crystals at various temperatures as shown in Fig. 1. The peak intensity decreases as temperature increases and change of the fine structure is observed. The atoms are distorted along the $c$-axis at tetragonal phase: There are three kinds of Ti-O atomic pairs; shorter Ti-O(1), medium $\mathrm{Ti}-\mathrm{O}(2)$ and longer $\mathrm{Ti}-\mathrm{O}(3)$ as shown in Fig. 3. The peaks from 1 to $2 \AA$ include three Ti-O pairs. In the case of Ti K-edge we tried to perform 3 shell-fitting in order to obtain the structural parameters and reasonable results were obtained. Our unknown parameters for the fitting are $r$ and $\sigma^{2}$ for each shell and the total number of them is 6 .

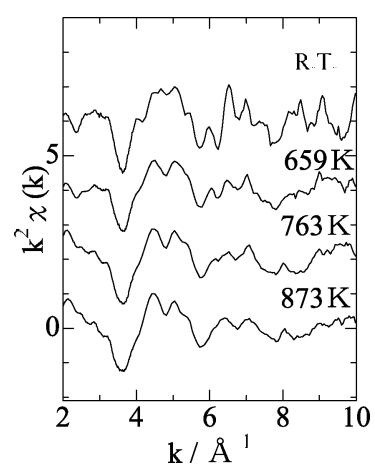

Fig. 1. EXAFS oscillation functions, $k^{2} \chi(k)$, of Ti K-edge EXAFS for $\mathrm{PbTiO}_{3}$ crystal at various temperatures. 


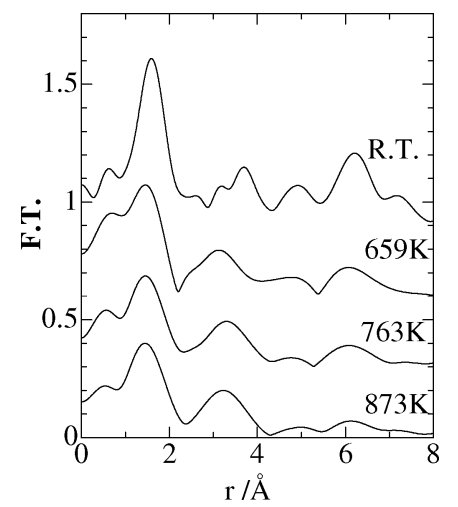

Fig. 2. Fourier transforms of $\mathrm{Ti} \mathrm{K}$-edge EXAFS for $\mathrm{PbTiO}_{3}$ crystals at various temperatures as shown in Fig. 1.

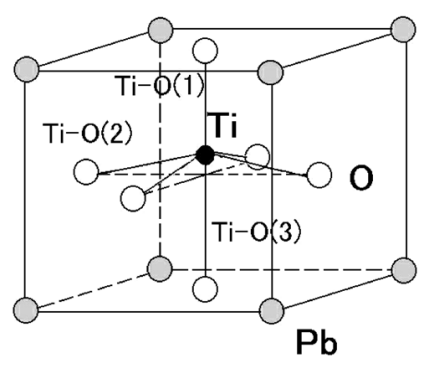

Fig. 3. Schematic structure of $\mathrm{PbTiO}_{3}$ in the tetragonal phase. The central atom is $\mathrm{Ti}$ (Black circles), white circles represent $\mathrm{O}$ atoms and gray circles represent $\mathrm{Pb}$ atoms.

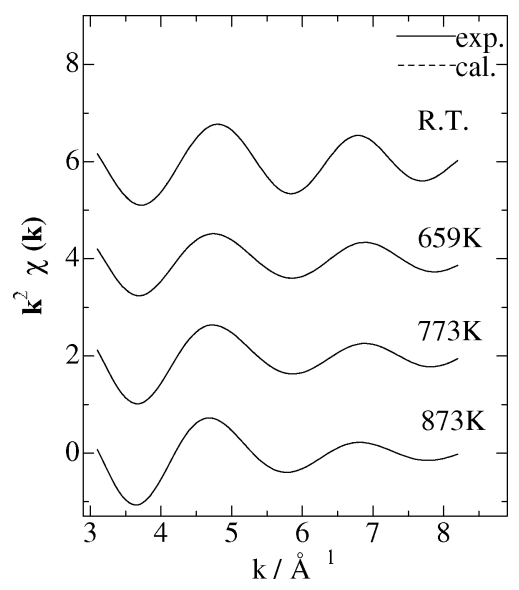

Fig. 4. Example of the quality for 3-shell fitting in the present study at various temperatures.

Figure 4 shows an example of the quality of 3 -shell fitting in the present study at various temperatures. The fitting results reproduce the experimental spectra well. Figure 5 shows the contributions of each shell as a function of $r$ which is obtained from the theoretical fitting for the Ti K-edge of $\mathrm{PbTiO}_{3}$. Figure 6 shows the variations of three kinds of Ti-O interatomic distances with temperature. We also easily find the tetragonal distortion below $T_{c}$ as shown in $\mathrm{Pb} \mathrm{L}_{\mathrm{III}}$-edge EXAFS [5]. For example in the tetragonal phase at $300 \mathrm{~K}$, the distance for $\mathrm{Ti}-\mathrm{O}(1)$ is about $1.8 \AA$, that for $\mathrm{Ti}-\mathrm{O}(2)$ is about $2.0 \AA$ and that for $\mathrm{Ti}-\mathrm{O}(3)$ is $2.4 \AA$. From these values we can estimate the distortion of $\mathrm{Ti}$ atoms with $\mathrm{O}$ atoms in the tetragonal phase. The distortion of $\mathrm{Ti}$ atoms from $\mathrm{O}$ atoms for $c$-axis is about $0.35 \AA$ at $300 \mathrm{~K}$ for the tetragonal phase, which is the same value as that obtained by Sicron et al. [3, 4].

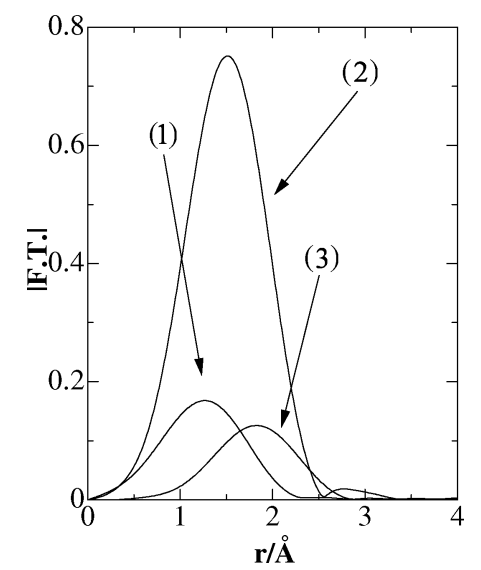

Fig. 5. The contributions of each shell in Ti K-edge fitting for $\mathrm{PbTiO}_{3}$ as a function of $r$ at $300 \mathrm{~K}$.

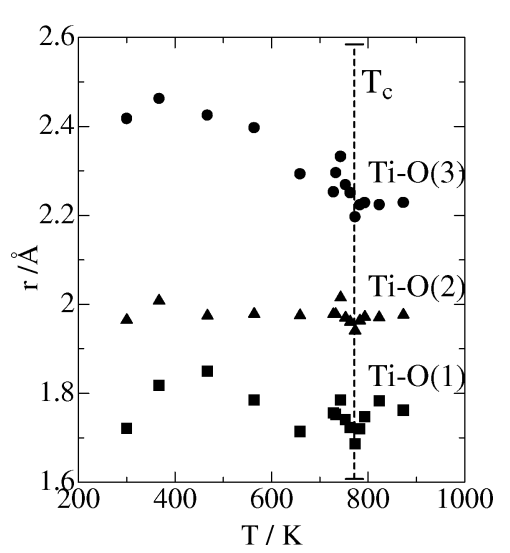

Fig. 6. Variations of three kinds of Ti-O interatomic distances with temperature.

The interatomic distance of $\mathrm{Ti}-\mathrm{O}(2)$ is almost constant in the whole temperature range including $T_{c}$. That of Ti-O(3) decreases as temperature increases up to $T_{c}$ and does not change with temperature at $T>T_{c}$. As for Ti-O(1), we find complex behavior between $\mathrm{Ti}-\mathrm{O}(2)$ and $\mathrm{Ti}-\mathrm{O}(3)$. That is: the longest $\mathrm{Ti}-\mathrm{O}(3)$ shows typical "displacive" behavior and Ti distortion remains at temperatures above $T_{c}$. These phenomena are quite similar to the result of $\mathrm{Pb}-\mathrm{O}$ obtained from $\mathrm{Pb} \mathrm{L}_{\mathrm{III}}$-edge EXAFS study [5]. At $T>T_{c}$, the interatomic distance for $\mathrm{Ti}-\mathrm{O}(1)$ is $1.75 \AA$, that for $\mathrm{Ti}-\mathrm{O}(2)$ is $2.0 \AA$, and that for $\mathrm{Ti}-\mathrm{O}(3)$ is $2.25 \AA$. This difference in Ti-O distance shows that atomic distortion remains at the cubic phase.

Figure 7 shows X-ray absorption near edge structure (XANES) of $\mathrm{Ti} \mathrm{K}$-edge for $\mathrm{PbTiO}_{3}$ at various temperatures. We can find two clear peaks (A and B) before the edge. The origins of these two peaks are assigned to the transition from $1 \mathrm{~s}$ to $3 \mathrm{~d}_{x y}$ and $3 \mathrm{~d}_{z}^{2}$ from the symmetry of the orbital, respectively. Although these transitions are forbidden generally in the dipole approximation, they are allowed by mixing with oxygen p-state orbitals due to the distortion of Ti atoms from the oxygen octahedron. That is the existence of these peaks indicates that $\mathrm{Ti}$ atoms are distorted.

Figure 8 shows the temperature variations of the intensity of peak A in Fig. 7 (solid circle). The peak intensity of A decreases gradually as the temperature increases up to $T_{c}$ and decreases discontinuously at $T_{c}$ and it is almost constant at $T>T_{c}$. The peak intensity of $\mathrm{A}\left(=I_{\mathrm{A}}\right)$ is known to be approximately proportional to the amplitude to the Ti atom distortion as [7],

$I_{A}=C e \cdot d$, 


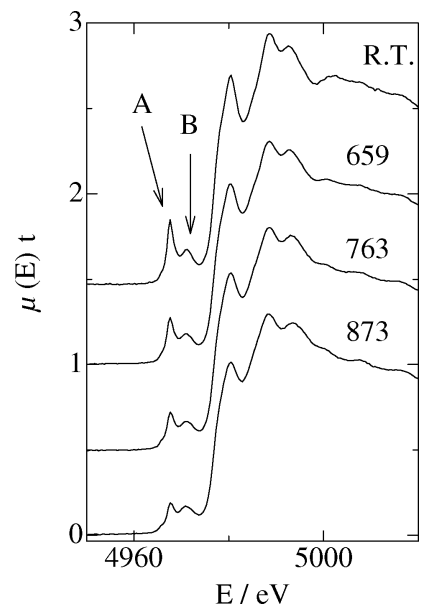

Fig. 7. X-ray absorption near edge (XANES) spectra of Ti K-edge for $\mathrm{PbTiO}_{3}$ at various temperatures.

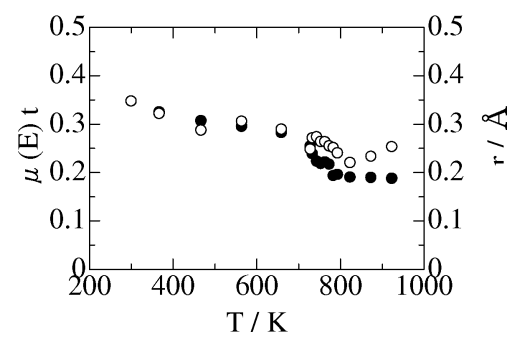

Fig. 8. Temperature variations of the intensity of peak A in XANES (Solid circle) and $\mathrm{Ti}$ atom distortion from the center of the oxygen octahedron (open circle).

where $\boldsymbol{e}$ and $d$ are the unit vector in the direction of the electric field of incident X-rays and the distortion of a Ti atom from the center of an oxygen octahedron, respectively. $C$ is a proportionality constant. The behavior in Fig. 8 indicates that the distortion of $\mathrm{Ti}$ atoms from $\mathrm{O}$ octahedron decreases as temperature increases and it remains finite at $T>T_{c}$. The values of the Ti atom distortion (d) along the $c$-axis with temperature evaluated from the present EXAFS by

$d=\left(r_{\mathrm{Ti}-\mathrm{O}(3)}-r_{\mathrm{Ti}-\mathrm{O}(1)}\right) / 2$

are also presented (open circle) in Fig. 8. When we put the constant $C$ to be 1.0 , the curve of $d$ vs $T$ coincides approximately with that of $I_{\mathrm{A}}$ vs $T$ at room temperature. $d$ shows the same behavior as $I_{A}$ and decreases as temperature increases up to $T_{c}$. As discussed previously $0.35 \AA$ of $d$ is the same as the value Sicron et al. reported [3, 4]. However they concluded that $d$ is almost constant at the temperature range from low temperature to $T_{c}$ and decreases slightly with $30 \%$ at $T>T_{c}$. On the other hand, our result shows clear decreasing of $d$ in the ferroelectric phase $\left(T<T_{c}\right)$.

\section{Conclusion}

We measured Ti K-edge EXAFS for $\mathrm{PbTiO}_{3}$ in the temperature range including $T_{c}$. The structural changes were discussed from the viewpoint of short-range order. Three kinds of atomic pairs, shorter Ti-O(1), medium Ti-O(2) and longer Ti-O(3) were analyzed independently. The longest $\mathrm{Ti}-\mathrm{O}(3)$ is discontinuously changed at the transition. The distortion of $\mathrm{Ti}$ atoms evaluated from EXAFS indicates that tetragonal distortion remains above $T_{c}$. The XANES study supports the temperature dependence the Ti distortion from EXAFS. The proposed solution may not be unique and further measurements, e.g. fluorescence mode for $\mathrm{Ti}$ K-edge XAFS, will hopefully confirm the obtained results.

\section{Acknowledgements}

The synchrotron radiation experiments were performed at Photon Factory in KEK under Proposal No. 2003G233.

\section{References}

1. Koningsberger, D. C. and Prins, R. (1998) "X-ray Absorption: Principles, Application Techniques of EXAFS, SEXAFS and XANES", (John Wiley and Sons Co, New York).

2. Shirane, G., Pepinsky, R. and Frazser, B. C., Acta Cryst. 9, 131 (1956).

3. Sicron, N., et al., Phys. Rev. B 50, 13168 (1994).

4. Ravel, B., et al., Ferroelectrics 164, 265 (1995).

5. Miyanaga, T., Diop, D., Ikeda, S. and Kon, H., Ferroelectrics 274, 41 (2002).

6. Sakane, H., et al., Jpn. J. Appl. Phys. 32, 4641 (1993).

7. Ravel, B., Stern, E. A. Vedrinski, R. I. and Kraizman, V., Ferroelectrics, 206-207, 407 (1998). 